radiotherapy, chemotherapy for SCLC), sixteen (37\%) patients with palliative intent (radiotherapy, chemotherapy, brachytherapy), and ten (23\%) patients received best supportive care (BSC) only. Of the five patients with PS 3, one received palliative radiotherapy and the other four BSC. Patients treated with curative intent had $71 \%$ survival at six months, $65 \%$ survival at 12 months: survival rates significantly higher compared with those receiving palliative treatment or BSC $(\mathrm{p}=0.02)$. There was no survival difference between palliative treatment and BSC ( $\mathrm{p}=$ 0.81) (Figure 1).

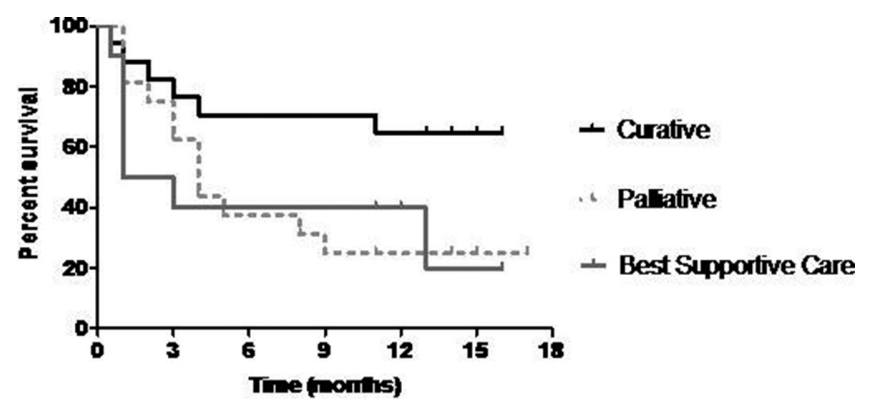

Abstract P170 Figure 1 Kaplan-Meier curves with survival stratified by treatment

Conclusion When a tissue diagnosis of lung cancer is pursued in those aged 75 and older, most patients will receive specific cancer treatment and this data informs clinical discussions about curative intent outcomes. In those who are PS 3 at baseline, BSC only is the likely outcome, and pursuing tissue diagnosis may not be appropriate.

\section{P171 A LOCAL CANCER NETWORK ROOT CAUSE AUDIT OF 62-DAY LUNG CANCER PATHWAY BREACHES}

${ }^{1} \mathrm{O}$ Eneje, ${ }^{1} \mathrm{~N}$ Kumar, ${ }^{2} \mathrm{D}$ Powrie, ${ }^{1} \mathrm{~B}$ Yung, ${ }^{3} \mathrm{M}$ Lawson. ${ }^{1}$ Basildon University Hospital NHS Foundation Trust, Basildon, UK; ${ }^{2}$ Southend University Hospital NHS Foundation Trust, Southend, UK; ${ }^{3}$ Broomfield Hospital, Chelmsford, UK

\subsection{6/thoraxjnl-2015-207770.308}

Introduction Over several years there has been an ongoing rise in 62-day Lung Cancer pathway referrals initiated by GPs as two-week wait referrals ( $2 \mathrm{WW})$. This was particularly marked in 2014 and breach rates increased across the East of England SCN. Within the Essex Lung Cancer Network an audit of these breaches was undertaken by three Trusts to look for common themes and to share best practice.

Methods Data were collected for all pathways that failed to meet the 62-day target across three NHS Trusts in Essex to identify any predictive factors for breaching the pathway. A standard proforma was used for abstraction. Results were analysed using GraphPad Prism 6 (La Jolla, CA).

Results In 2014 a total of 1,419 2WW referrals were received by the three Trusts of which $13-23 \%$ were diagnosed with lung cancer. Between 19\% and 54\% breached the 62-day target (89 of 246 pathways). The median length of the breached pathways varied from $86-88$ days by Trust. Trusts did not appear to differ significantly by end treatment after pathway breach. There were generic common themes within the breached pathways of each Trust but for the two worst performing Trusts specific pathway issues were identified. In one Trust it was clear that time delays to perform CT guided lung biopsies with a 2.75 relative risk of breaching if a pathway involved a CT biopsy (95\% CI 1.6-4.6, $\mathrm{p}<0.0001)$. At another Trust a high proportion of breached pathways had a bronchoscopy as the first test but went on to have further diagnostic biopsies by other methods.

Conclusion Many of the diagnostic delays were due to complex patient pathways needing multiple diagnostic tests. However for two Trusts significant problems were highlighted for targeted quality improvement plans. Selecting the best test to give diagnostic and staging information is vital particularly when services are stretched and capacity is reached.

\section{P172 TRAINING NURSES IN SAMPLING AND ACQUISITION OF SPECIMEN DURING EBUS GUIDED TRANSBRONCHIAL NEEDLE ASPIRATION}

V Johnson, W Stables, S Binu, C Smyth, M Walshaw, K Mohan. Liverpool Heart and Chest Hospital, Liverpool, UK

10.1136/thoraxjnl-2015-207770.309

Introduction EBUS has now become the standard of choice for mediastinal staging and in the diagnosis of paratracheal and peribronchial lesions. EBUS is usually performed as a 2 person procedure, one to locate and align the bronchoscope and a second to carry out node sampling. Whilst these have traditionally both been medical personnel, with the increase in use of this procedure and alterations in the training and availability of medical staff, we wished to look at the feasibility of empowering nurses to perform needle aspiration during EBUS.

Methods We provide a regional service for EBUS, carrying out over 420 procedures per year, often in technically difficult cases where local hospital EBUS has been unhelpful. From January 2015 we trained a band 5 registered nurse in needle aspiration, who randomly assisted in the procedure in 93 of 172 cases up to June 2015. We report our experience in these 93 cases.

Results These 93 patients were referred from 12 hospitals and 20 had undergone previous undiagnostic procedures (6 EBUS, 14 bronchoscopy). The mean age was 67 years (range $27-87$ ) and 50 were male. 82 were performed under local anaesthesia using lignocaine spray and intravenous midazolam (1 - $10 \mathrm{mg}$; mean $4 \mathrm{mgs}$ ) and the remaining 11 under general anaesthesia. 200 lymph nodes (2R, 4R, 4L, 7, 10R, 10L, 11R) and 13 lung lesions were biopsied. Results were as follows: Adequate samples were obtained in 99\% (91/93) and the NSCLC - NOS rate was 2\%. 31 adenocarcinoma, 10 squamous cell carcinoma, 10 small cell carcinoma, 1 NSCLC - NOS, 1 large cell neuroendocrine carcinoma, 1 soft tissue lesion (repeat EBUS showed myxoid spindle cell mesenchymal lesion), 1 breast carcinoma, 19 nonspecific benign nodes, 15 sarcoid and 2 TB. There were no complications.

Conclusion Our findings suggest that with motivated staff and adequate training, it is possible for nurses to perform needle aspiration during EBUS procedure with excellent results. This could be adopted as the method of choice by other centres as EBUS services continue to expand.

\section{P173 THE INVESTIGATION OF PATIENTS REFERRED WITH HAEMOPTYSIS VIA THE TWO WEEK WAIT SYSTEM IN SOUTHMEAD HOSPITAL, BRISTOL, UK}

J Harper, J Curran, M Plummeridge. Southmead Hospital, Bristol, UK

10.1136/thoraxjnl-2015-207770.310 
Background Southmead Hospital is a large teaching Hospital in the South West of England. GP two week wait (TWW) referrals to the respiratory department are sent via a standardised proforma with one of a number of reasons for referral selected. We conducted this project both to evaluate how we are currently investigating patients referred with haemoptysis and to identify whether our diagnostic pathway for patients for these patients could be optimised. We were particularly interested as to whether bronchoscopy is diagnostically helpful in this cohort of patients.

Methods We looked at all TWW referrals between 13/6/13 and 2/4/15 (825 referrals) and selected those who were referred for haemoptysis (110 patients). The clinical course of these patients was tracked retrospectively by looking at our electronic record system and clinical letters.

Results Overall 109 of the 110 patients identified had a CT scan - this was normal in 31 patients. In the remainder of cases the CT was suggestive of malignancy in 38 of the 78 abnormal scans. The remainder of CT scans had positive findings that were not suggestive of malignancy. Of the 31 patients who had a normal scan, 22 patients underwent bronchoscopy. 40 patients had abnormal CT scan that were not suggestive of malignancy. 25 of these patients went on to have bronchoscopy All bronchoscopies were either normal or showed non specific findings.16 patients who had an eventual diagnosis of lung malignancy had an initial CT scan suggestive of malignancy.

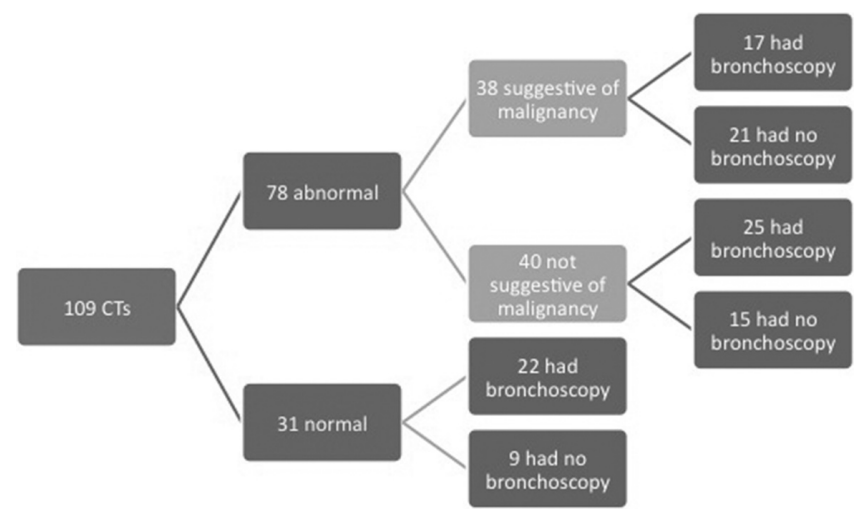

Abstract P173 Figure 1

Conclusion Our project demonstrates that currently 58\% of patients referred with haemoptysis via the TWW system go on to have a bonchoscopy. In our cohort of patients all bronchoscopies were either normal or showed non-specific changes. All patients with lung malignancy had a prior CT that was suggestive of malignancy and did not require a bronchoscopy other than as a potential means of obtaining tissue. We suggest that bronchoscopy may not be necessary in patients referred with haemoptysis who have a normal CT scan. We feel this will change our local practice and may enable us to better target this investigation to patients who will benefit from it.

\section{P174 CATCH - A YEAR IN PROFILE AND FURTHER REDUCTIONS IN 2WW REFERRALS}

${ }^{1} \mathrm{VTY}$ Ng, ${ }^{2} \mathrm{~A}$ Walsham, ${ }^{2} \mathrm{~A}$ Sharman, ${ }^{2} \mathrm{SCO}$ Taggart. ${ }^{1}$ Manchester University Medical School, Manchester, UK; ${ }^{2}$ Salford Royal NHS Foundation Trust, Salford, UK

10.1136/thoraxjnl-2015-207770.311
Introduction In Salford, annual 2WW referrals rose from 235 in 2010/11 to $248(2011 / 12)$ and $281(2012 / 13)$ but fell to 249 in $2013 / 14$ as the result of a 5 month pilot ${ }^{1}$ of our CATCH protocol (Community Access To CT Chest) allowing abnormal "low risk" CXR reports to trigger a GP request for a fast track CT scan. This audit reviews the performance of CATCH for a whole year of activity from $1^{\text {st }}$ May 2014 to $30^{\text {th }}$ April 2015.

Methods The CATCH d-base and electronic patient record were used to identify the patients and dates of CXR and CT examinations in addition to CXR/CT scan reports and final diagnoses. The number of $2 \mathrm{WW}$ referrals was determined for the same time period using the cancer waiting times d-base.

Results A total of 117 patients entered the CATCH protocol of which the majority of CXRs demonstrated the presence of a well-defined (47\%) or ill-defined opacity (14\%) and a further $18 \%$ revealed abnormality at the hilum. The remaining CXRs (21\%) raised concerns about fat pads, atelectasis or pleural abnormality. For the 115 patients having a CT scan, the findings confirmed cancer in 9\%, solitary pulmonary nodule (25\%), infection/inflammation (15\%), atelectasis (10\%), pleural plaque $(10 \%)$, fat pad $(5 \%)$ and in $11 \%$ the CT scan was normal.

Following CATCH CT scan, 53 (46\%) patients required no follow up, 33 (29\%) generated urgent referral, 16 (14\%) nonurgent referral to the chest clinic, $11(10 \%)$ required follow up surveillance imaging. Timelines for $\mathrm{CATCH}$ management are detailed in Table 1. Mean time from CT report to cancer diagnosis was 61.1 days (range 23 to 187 ) and total number of $2 \mathrm{WW}$ referrals for 2014/15 was 234 .

\begin{tabular}{ll} 
Abstract P174 Table 1 & Mean time for main CATCH outcomes \\
\hline CXR performed to CXR report & 4.0 days \\
CXR report to CT appointment & 14.6 days \\
CT appointment to CT report & 0.8 days \\
\hline
\end{tabular}

Conclusions Following the introduction of CATCH to the Salford Lung Cancer Service, $2 \mathrm{WW}$ referrals have fallen further to manageable numbers. The pick-up rate for cancer is only small and reflects the low risk abnormality detected on CXR. The relatively long diagnostic times for cancer reflect the processing of small nodules detected within this select group of patients.

\section{REFERENCE}

1 Moorcroft CS, Kamalatharen G, Elliot S, et al. CATCH - community access to CT chest. Thorax 2014;69:A115 doi:10.1136/thoraxjnl-2014-206260.231

\section{P175 USE OF A VIRTUAL CLINIC TO IMPROVE THE LUNG CANCER PATIENT JOURNEY}

A Nanapragasam, N Maddock, A Mclver, C Smyth, MJ Walshaw. Liverpool Heart and Chest Hospital, Liverpool, UK

\subsection{6/thoraxjnl-2015-207770.312}

In 2014 with our primary care colleagues we introduced a 'Straight to CT' system for out-patients with a radiological or clinical suspicion of lung cancer. The CT was done on behalf of primary care, and only patients who had a CT suspicious of lung cancer, were automatically taken by the lung cancer team. Such scans are reviewed by a cancer clinician, who makes a provisional next best test plan, and this empowers a telephone clerking by a highly specialised lung cancer nurse. The assessment 